\title{
ESTIMATES FOR THE MAXIMAL CAUCHY INTEGRAL ON CHORD-ARC CURVES
}

\author{
Carmelo Puliatti \\ Universitat Autònoma de Barcelona, BGSMath and Departament de Matemàtiques \\ 08193, Bellaterra, Barcelona, Catalonia; puliatti@mat.uab.cat
}

\begin{abstract}
We study the chord-arc Jordan curves that satisfy the Cotlar-type inequality $T_{*}(f) \lesssim M^{2}(T f)$, where $T$ is the Cauchy transform, $T_{*}$ is the maximal Cauchy transform and $M$ is the Hardy-Littlewood maximal function. Under the background assumption of asymptotic conformality we find a characterization of such curves in terms of the smoothness of a parametrization of the curve.
\end{abstract}

\section{Introduction}

Consider a homogeneous smooth Calderón-Zygmund operator in $\mathbf{R}^{n}$

$$
T f(x)=\text { p.v. } \int f(x-y) K(y) d y \equiv \lim _{\epsilon \rightarrow 0} T_{\epsilon} f(x), \quad x \in \mathbf{R}^{n},
$$

where $T_{\epsilon}$ is the truncation at level $\epsilon$ defined by

$$
T_{\epsilon} f(x)=\int_{|y|>\epsilon} f(x-y) K(y) d y, \quad x \in \mathbf{R}^{n},
$$

and $f$ is in $L^{p}\left(\mathbf{R}^{n}\right), 1 \leq p<\infty$. Here the kernel $K$ is of class $C^{\infty}$ off the origin, homogeneous of order $-n$ and with zero integral on the unit sphere

$$
\left\{x \in \mathbf{R}^{n}:|x|=1\right\} \text {. }
$$

Let $T_{*}$ be the maximal singular integral

$$
T_{*} f(x)=\sup _{\epsilon>0}\left|T_{\epsilon} f(x)\right|, \quad x \in \mathbf{R}^{n} .
$$

A classical fact relating $T_{*}$ and the standard Hardy-Littlewood maximal operator $M$ is Cotlar's inequality, which reads

$$
T_{*}(f)(x) \leq C(M(T f)(x)+M(f)(x)), \quad x \in \mathbf{R}^{n} .
$$

Combining this with the $L^{p}$ estimates $\|T(f)\|_{p} \leq C\|f\|_{p}$ and $\|M(f)\|_{p} \leq C\|f\|_{p}$, $1<p<\infty$ one gets $\left\|T_{*}(f)\right\|_{p} \leq C\|f\|_{p}, 1<p<\infty$.

It was discovered in [4] that if $T$ is an even higher order Riesz transform, that is, if $K(x)=P(x) /|x|^{n+d}$, with $P$ an even homogeneous polynomial of degree $d$, then one can get rid of the second term in the right hand side of (1.1), namely,

$$
T_{*}(f)(x) \leq C M(T f)(x), \quad x \in \mathbf{R}^{n} .
$$

https://doi.org/10.5186/aasfm.2018.4362

2010 Mathematics Subject Classification: Primary 42B20, 30C62, 28A80.

Key words: Cauchy integral, Cotlar's inequality, asymptotically conformal curve, chord-arc curve. 
Hence $\left\|T_{*}(f)\right\|_{p} \leq C\|T(f)\|_{p}, 1<p<\infty$, in this case. However, if $T$ is an odd higher order Riesz transform, then (1.2) may fail and the right substitute turns out to be (see [3])

$$
T_{*}(f)(x) \leq C M^{2}(T f)(x), \quad x \in \mathbf{R}^{n},
$$

where $M^{2}$ stands for the iteration of $M$.

Inequalities of the type (1.2) and (1.3) were first considered in relation to the David-Semmes problem (see [4],[3] and [9]) and later on were studied in the context of the Cauchy singular integral on Lipschitz graphs and $C^{1}$ curves by Girela-Sarríon in [2]. Let $\Gamma$ be either a Lipschitz graph or a closed chord-arc curve in the plane, let $T$ be the Cauchy Singular Integral and $M$ the Hardy-Littlewood maximal operator, both with respect to the arc-length measure, and let $T_{*}$ be the maximal Cauchy Integral. Precise definitions will be given below. Girela-Sarrión showed in [2] that the presence at a point $z$ of the curve of a non-zero angle prevents (1.3), with $x$ replaced by $z$, to hold. This agrees with the intuition that (1.3) should help in finding tangent lines, but suggests that it is a condition definetely stronger than the mere existence of tangents. It was also shown in [2] that if $\Gamma$ is a closed $C^{1}$ curve with the property that the modulus of continuity $\omega(z, \delta)$ of the unit tangent vector satisfies

$$
\omega(z, \delta) \leq C \frac{1}{\log \left(\frac{1}{\delta}\right)}, \quad z \in \Gamma, \quad \delta<1 / 2,
$$

then (1.3) holds with $x \in \mathbf{R}^{n}$ replaced by $z \in \Gamma$. Observe that condition (1.4) quantifies the absence of corners in a curve for which (1.3) holds. In this paper we study the validity of inequality (1.3) in the context of chord-arc curves. A chord-arc curve is a rectifiable Jordan curve $\Gamma$ in the plane with the property that there exists a positive constant $C$ such that, given any two points $z_{1}, z_{2} \in \Gamma$ one has

$$
l\left(z_{1}, z_{2}\right) \leq C\left|z_{1}-z_{2}\right|,
$$

where $l\left(z_{1}, z_{2}\right)$ is the length of the shortest arc in $\Gamma$ joining $z_{1}$ and $z_{2}$. Equivalently $\Gamma$ is a bilipschitz image of the unit circle (see [5], Theorem 7.9). Then $\Gamma$ can be parametrized by a periodic function $\gamma: \mathbf{R} \rightarrow \Gamma$ of period $T$ satisfying the bilipschitz condition

$$
\frac{1}{L}|x-y| \leq|\gamma(x)-\gamma(y)| \leq L|x-y|, \quad x, y \in \mathbf{R}, \quad|x-y| \leq \frac{T}{2},
$$

for some positive constant $L$. We say, by slightly abusing language, that $\gamma$ is a bilipschitz parametrization of $\Gamma$. One can take, for instance, the $T$-periodic extension of the arc-length parametrization of $\Gamma$ with $T$ being the length of $\Gamma$.

One can easily define the maximal Hardy-Littlewood operator and the Cauchy Integral on a chord-arc curve. Given $z \in \Gamma$ let $t \in \mathbf{R}$ be such that $z=\gamma(t)$. Set

$$
\Gamma_{z, r}:=\gamma(\{\tau:|\tau-t|<r\}) .
$$

One should look at $\Gamma_{z, r}$ as "balls" of radius $r$ centered at $z$. Indeed, owing to the bilipschitz condition (1.5), each $\Gamma_{z, r}$ contains and is contained in a disc in $\Gamma$ of radius comparable to $r$, for $r<T$. It will be more convenient to work with $\Gamma_{z, r}$ than with the euclidean discs $D(z, r) \cap \Gamma$, where $D(z, r)$ stands for the planar disc of center $z$ and radius $r$. 
Denote by $\mu$ the arc-length measure on $\Gamma$. For $f \in L^{1}(\Gamma, \mu)$ and $z \in \Gamma$, we define the Hardy-Littlewood maximal function on the curve $\Gamma$ as

$$
M f(z):=\sup _{r>0} \frac{1}{\mu\left(\Gamma_{z, r}\right)} \int_{\Gamma_{z, r}}|f| d \mu .
$$

The Cauchy Integral is defined as

$$
T f(z)=\mathrm{p} \cdot \mathrm{v} \cdot \frac{1}{\pi i} \int_{\Gamma} \frac{1}{w-z} f(w) d w \equiv \lim _{\epsilon \rightarrow 0} T_{\epsilon} f(z), \quad z \in \Gamma,
$$

where

$$
T_{\epsilon} f(z)=\frac{1}{\pi i} \int_{\Gamma \backslash \Gamma_{z, \epsilon}} \frac{f(w)}{w-z} d w
$$

is the truncated Cauchy Integral at level $\epsilon$. The maximal Cauchy Integral is

$$
T_{*} f(z):=\sup _{\epsilon>0}\left|T_{\epsilon} f(z)\right| .
$$

Our aim is to investigate under what conditions on $\Gamma$ one has the inequality

$$
T_{*} f(z) \leq C M^{2}(T f)(z), \quad z \in \Gamma, \quad f \in L^{2}(\Gamma, \mu),
$$

where $C$ is a positive constant. Since we know that angles prevent the above inequality to hold, we need to require on $\Gamma$ a condition that excludes them. One such a condition is asymptotic conformality. Given two points $z_{1}, z_{2} \in \Gamma$ let $A\left(z_{1}, z_{2}\right)$ be the arc in $\Gamma$ joining the two points and having smallest diameter (there is only one if the two points are sufficiently close). The Jordan curve $\Gamma$ is said to be asymptotically conformal if, given a positive number $\delta$ there exists a positive $\epsilon$, so that for any two points $z_{1}, z_{2} \in \Gamma$ satisfying $\left|z_{1}-z_{2}\right|<\epsilon$ one has

$$
\left|z_{1}-z\right|+\left|z_{2}-z\right| \leq(1+\delta)\left|z_{1}-z_{2}\right|, \quad z \in A\left(z_{1}, z_{2}\right)
$$

Our main result reads as follows.

Theorem. Let $T$ be the Cauchy Integral on an asymptotically conformal chordarc curve $\Gamma$ and let $\gamma$ be a bilipschitz parametrization of $\Gamma$. Then the estimate

$$
T_{*}(f)(z) \leq C M^{2}(T f)(z), \quad z \in \Gamma, \quad f \in L^{2}(\Gamma, \mu),
$$

holds if and only if there exists $C>0$ such that

$$
|\gamma(x+\epsilon)+\gamma(x-\epsilon)-2 \gamma(x)| \leq C \frac{\epsilon}{|\log \epsilon|}
$$

for each $\epsilon$ satisfying $0<\epsilon<T$ and for each $x \in \mathbf{R}$.

One should recall that condition (1.7) implies that $\gamma$ is differentiable almost everywhere in the ordinary sense and the derivative is a function of vanishing mean oscillation (see [11]). Therefore, for chord arc curves satisfying the background assumption of asymptotical conformality, inequality (1.6) is equivalent to the precise form of differentiability described in terms of second order differences in (1.7). Also notice that if $\gamma$ is the arc-length parametrization of a $C^{1}$ curve, (1.4) implies (1.7), so that the Theorem generalizes Girela-Sarrión's result.

In Section 2 we prove a couple of Lemmas which allow to express condition (1.6) in an equivalent form in terms of a function related to the geometry of $\Gamma$. Section 3 is devoted to take care of a technical question, namely, that it is enough to estimate truncations at small enough levels. In Section 4 we prove the Theorem by means of three lemmas, one on them making the connection between the function carrying the geometrical information and the second difference condition (1.7). In Section 5 
we present an example of a spiraling domain that enjoys the equivalent conditions in the Theorem but whose boundary is not of class $C^{1}$. This example justifies the efforts made in order to extend the condition (1.4) to a less regular case since new geometric behaviors can be detected.

Our terminology and notation are standard. We let $C$ denote a constant independent of the relevant variables under consideration and which may vary at each occurrence. The notation $A \lesssim B$ means that there exists a constant $C>0$ such that $A \leq C B$. We write $A \gtrsim B$ if $B \lesssim A$. The disc centered at $z$ of radius $r$ is denoted by $D(z, r)$.

\section{Two preliminary lemmas}

The beginning of the proof follows the ideas of [2], so that we will be rather concise. Given a function $f \in L^{1}(\Gamma, \mu)$ we denote by $m_{\Gamma_{z, \epsilon}}(f)=f_{\Gamma_{z, \epsilon}} f(w) d \mu(w)$ the mean of $f$ on $\Gamma_{z, \epsilon}$ with respect to the arc length measure $\mu$. We let $K_{z, \epsilon}$ denote the Cauchy kernel truncated at the point $z$ at level $\epsilon$, that is,

$$
K_{z, \epsilon}(w)=\frac{1}{\pi i} \frac{1}{w-z} \chi_{\Gamma \backslash \Gamma_{z, \epsilon}}(w), \quad w \in \Gamma .
$$

Set $g_{z, \epsilon}=T\left(K_{z, \epsilon}\right)$ and let $N>1$ be a big number to be chosen later. Following [2, p. 673] we obtain the identity

$$
-T_{\epsilon} f(z)=I_{\epsilon}+I I_{\epsilon}+I I I_{\epsilon}
$$

where

$$
\begin{aligned}
I_{\epsilon} & :=\int_{\Gamma_{z, N \epsilon}} \operatorname{Tf}(w)\left(g_{z, \epsilon}(w)-m_{\Gamma_{z, N \epsilon}}\left(g_{z, \epsilon}\right)\right) d w \\
I I_{\epsilon} & :=m_{\Gamma_{z, N \epsilon}}\left(g_{z, \epsilon}\right) \int_{\Gamma_{z, N \epsilon}} T f(w) d w \\
I I I_{\epsilon} & :=\int_{\Gamma \backslash \Gamma_{z, N \epsilon}} T f(w) g_{z, \epsilon}(w) d w .
\end{aligned}
$$

Following closely the argument in [2] one can prove that

$$
\begin{aligned}
\left|I_{\epsilon}\right| & \leq C M^{2}(T f)(z), \\
\left|I I_{\epsilon}\right| & \leq C M(T f)(z),
\end{aligned}
$$

where the constant $C$ does not depend on the choice of $N$. Since clearly $M(g) \leq$ $M^{2}(g)$ for any $g$, we are left with the task of estimating $I I I_{\epsilon}$. The next lemma provides an expression for $I I I_{\epsilon}$ in terms of a function encoding the smoothness of $\Gamma$. To state the lemma first we need to clarify the definition of a branch of the logarithm of $w-z$, as a function of $w$ with $z \in \Gamma$ fixed, in an appropriate region.

Given $z \in \Gamma$ let $\Delta_{z}$ be a curve connecting $z$ and $\infty$ in the unbounded component of $\mathbf{C} \backslash \Gamma$. Such curves exist and indeed we will construct a special one in Section 4 (under the additional assumption of asymptotic conformality). Hence $\mathbf{C} \backslash \Delta_{z}$ is a simply connected domain containing $\Gamma \backslash\{z\}$ and so there exists in $\mathbf{C} \backslash \Delta_{z}$ a branch of $\log (w-z)$. In particular, if $z=\gamma(x)$ for some $x \in \mathbf{R}$, the expressions $\log (\gamma(x+\epsilon)-\gamma(x))$ and $\log (\gamma(x-\epsilon)-\gamma(x))$ make sense for $0<\epsilon<T$.

Lemma 1. Let $\Gamma$ be a chord-arc curve and $\gamma$ a bilipschitz parametrization of $\Gamma$. Let $z \in \Gamma$ and let $x$ be a real number such that $\gamma(x)=z$. Then for almost every 
$w \in \Gamma \backslash \Gamma_{z, N \epsilon}$ we have

$$
T\left(K_{z, \epsilon}\right)(w)=\frac{1}{\pi^{2}(z-w)}\left[F(x, \epsilon)+G_{z, \epsilon}(w)\right],
$$

where

$$
F(x, \epsilon)=\log (\gamma(x+\epsilon)-\gamma(x))-\log (\gamma(x-\epsilon)-\gamma(x))+\pi i
$$

and

$$
\left|G_{z, \epsilon}(w)\right| \leq \frac{C \epsilon}{|z-w|}
$$

Proof. Take $w \in \Gamma \backslash \Gamma_{z, N \epsilon}$. Then

$$
\begin{aligned}
T\left(K_{z, \epsilon}\right)(w) & =-\frac{1}{\pi^{2}} \lim _{\delta \rightarrow 0} \int_{\Gamma \backslash\left(\Gamma_{w, \delta} \cup \Gamma_{z, \epsilon}\right)} \frac{1}{(\zeta-z)(\zeta-w)} d \zeta \\
& =-\frac{1}{\pi^{2}} \frac{1}{w-z} \lim _{\delta \rightarrow 0} \int_{\Gamma \backslash\left(\Gamma_{w, \delta} \cup \Gamma_{z, \epsilon}\right)}\left(\frac{1}{\zeta-w}-\frac{1}{\zeta-z}\right) d \zeta .
\end{aligned}
$$

Let $y \in \mathbf{R}$ with $\gamma(y)=w$. Then the latest integral in the above formula is

$$
\begin{aligned}
& \log (\gamma(y-\delta)-\gamma(y))-\log (\gamma(x+\epsilon)-\gamma(y))+\log (\gamma(x-\epsilon)-\gamma(y)) \\
& -\log (\gamma(y+\delta)-\gamma(y))-(\log (\gamma(y-\delta)-\gamma(x)) \\
& -\log (\gamma(x+\epsilon)-\gamma(x))+\log (\gamma(x-\epsilon)-\gamma(x))-\log (\gamma(y+\delta)-\gamma(x))) .
\end{aligned}
$$

Assume that $\gamma$ is differentiable at the point $y$ and the derivative $\gamma^{\prime}(y)$ does not vanish. Then we have that

$$
\lim _{\delta \rightarrow 0}(\log (\gamma(y-\delta)-\gamma(y))-\log (\gamma(y+\delta)-\gamma(y)))=\pi i,
$$

because the curve $\Delta_{w}$ lies in the unbounded component of $\mathbf{C} \backslash \Gamma$, and then to the right hand side of $\Gamma$, oriented according to the parametrization $\gamma$. Taking limit as $\delta$ goes to 0 we obtain

$$
\begin{aligned}
T\left(K_{z, \epsilon}\right)(w)= & -\frac{1}{\pi^{2}} \frac{1}{w-z}((\log (\gamma(x+\epsilon)-\gamma(x))-\log (\gamma(x-\epsilon)-\gamma(x))+\pi i) \\
& -(\log (\gamma(x+\epsilon)-\gamma(y))-\log (\gamma(x-\epsilon)-\gamma(y)))) .
\end{aligned}
$$

Define

$$
G_{z, \epsilon}(w)=\log (\gamma(x-\epsilon)-\gamma(y))-\log (\gamma(x+\epsilon)-\gamma(y)) .
$$

It remains to show the decay inequality (2.2). According to the choice of $\Delta_{w}$ we have a well defined branch of $\log (\gamma(x+t)-w),-\epsilon<t<\epsilon$. Thus

$$
G_{z, \epsilon}(w)=-\int_{-\epsilon}^{\epsilon} \frac{d}{d t} \log (\gamma(x+t)-w) d t=-\int_{-\epsilon}^{\epsilon} \frac{\gamma^{\prime}(x+t)}{\gamma(x+t)-w} d t .
$$

Since $w=\gamma(y) \in \Gamma \backslash \Gamma_{z, N \epsilon}$, we have $y \notin(x-N \epsilon, x+N \epsilon)$ and so

$$
|w-z|=|\gamma(y)-\gamma(x)| \geq \frac{|y-x|}{L} \geq \frac{N \epsilon}{L},
$$

which gives, taking $N \geq 2 L^{2}$,

$$
\begin{aligned}
|w-\gamma(x+t)| & \geq|w-z|-|\gamma(x)-\gamma(x+t)| \\
& \geq \frac{|w-z|}{2}+\frac{N \epsilon}{2 L}-L \epsilon \geq \frac{|w-z|}{2} .
\end{aligned}
$$


Hence, by (2.3),

$$
\left|G_{z, \epsilon}(w)\right| \leq \int_{-\epsilon}^{\epsilon} \frac{\left|\gamma^{\prime}(x+t)\right|}{|\gamma(x+t)-w|} d t \leq \frac{4 L \epsilon}{|w-z|} .
$$

Lemma 2. Let $\Gamma$ be a chord-arc curve and $\gamma$ a bilipschitz parametrization of $\Gamma$. Then the inequality

$$
T_{*}(f)(z) \leq C M^{2}(T f)(z), \quad z \in \Gamma, \quad f \in L^{2}(\Gamma, \mu),
$$

is equivalent to

$$
|F(x, \epsilon)||\log (\epsilon)| \leq C, \quad 0<\epsilon<T, \quad x \in \mathbf{R} .
$$

Proof. Assume that (2.5) holds. Then by Lemma 1

$$
\begin{aligned}
I I I_{\epsilon} & =\int_{\Gamma \backslash \Gamma_{z, N \epsilon}} T f(w) T\left(K_{z, \epsilon}\right)(w) d w \\
& =\frac{F(x, \epsilon)}{\pi^{2}} \int_{\Gamma \backslash \Gamma_{z, N \epsilon}} \frac{T f(w)}{z-w} d w+\frac{1}{\pi^{2}} \int_{\Gamma \backslash \Gamma_{z, N \epsilon}} T f(w) \frac{G_{z, \epsilon}(w)}{z-w} d w \\
& =F(x, \epsilon) I V_{\epsilon}+V_{\epsilon},
\end{aligned}
$$

where the last identity is a definition of the terms $I V_{\epsilon}$ and $V_{\epsilon}$. One can break the domain of integration in the integrals in $I V_{\epsilon}$ and $V_{\epsilon}$ into a union of dyadic annuli

$$
A_{j}=\gamma\left\{y \in \mathbf{R}: N \epsilon 2^{j}<|y-x| \leq N \epsilon 2^{j+1}\right\}, \quad j=0,1, \ldots,
$$

then perform standard estimates and apply (2.2) to get, thanks to the quadratic decay of the integrand,

$$
\left|V_{\epsilon}\right| \leq C M(T(f))(z)
$$

For $I V_{\epsilon}$ one only has a first order decay, which gives

$$
\left|I V_{\epsilon}\right| \leq C\left|\log \left(\frac{N L}{\epsilon}\right)\right| M(T f)(z),
$$

thus completing the proof of the sufficient condition.

Assume now (2.4). Recalling that $I I I_{\epsilon}=F(x, \epsilon) I V_{\epsilon}+V_{\epsilon}$ and (2.6), we obtain

$$
\left|F(x, \epsilon) I V_{\epsilon}\right| \leq C M^{2}(T(f))(z), \quad z \in \Gamma, \quad f \in L^{2}(\Gamma, \mu) .
$$

The Cauchy Singular Integral operator $T$ is an isomorphism of $L^{2}(\Gamma, \mu)$ onto itself. This is proved in Lemma 1 of $[2$, p. 661] for Lipschitz graphs, and the same proof works in our context. Thus (2.7) can be rewritten as

$$
\left|F(x, \epsilon) \int_{\Gamma \backslash \Gamma_{z, N \epsilon}} \frac{g(w)}{z-w} d w\right| \leq C M^{2}(g)(z), \quad z \in \Gamma, \quad g \in L^{2}(\Gamma, \mu) .
$$

To simplify the notation take $x=0=\gamma(x)$. Assume first that $0<\epsilon<1$. Apply (2.8) with $g$ the characteristic function of $\gamma\left(\left(\epsilon^{n}, \epsilon\right)\right)$, where $n$ is a large integer to be chosen. Then

$$
|F(0, \epsilon)|\left|\int_{\epsilon^{n}}^{\epsilon} \frac{\gamma^{\prime}(t)}{\gamma(t)} d t\right| \leq C
$$


and

$$
\begin{aligned}
\left|\int_{\epsilon^{n}}^{\epsilon} \frac{\gamma^{\prime}(t)}{\gamma(t)} d t\right| & =\left|\log (\gamma(\epsilon))-\log \left(\gamma\left(\epsilon^{n}\right)\right)\right| \\
& \geq\left|\log (|\gamma(\epsilon)|)-\log \left(\left|\gamma\left(\epsilon^{n}\right)\right|\right)\right| \geq \log \left(\frac{1}{L^{2} \epsilon^{n-1}}\right) \\
& \geq-2 \log (L)+(n-2) \log \left(\frac{1}{\epsilon}\right)+\log \left(\frac{1}{\epsilon}\right) \geq|\log (\epsilon)|
\end{aligned}
$$

provided $n=n(\epsilon)$ is large enough so that $-2 \log (L)+(n-2) \log (1 / \epsilon) \geq 0$. Therefore (2.5) follows in this case.

If $1 \leq \epsilon<T$ then we take as $g$ the characteristic function of $\gamma\left(\left(\epsilon^{-n}, \epsilon\right)\right)$. In this case we get

$$
\left|\int_{\epsilon^{-n}}^{\epsilon} \frac{\gamma^{\prime}(t)}{\gamma(t)} d t\right| \geq-2 \log (L)+n \log (\epsilon)+\log (\epsilon) \geq|\log (\epsilon)|
$$

provided $n$ is chosen so that $-2 \log (L)+n \log (\epsilon) \geq 0$.

\section{Reduction to estimating truncations at small levels}

In this section we reduce the proof of (1.6) to estimating the truncations $T_{\epsilon} f$ for small $\epsilon$. In the previous section we showed that the estimate of $T_{\epsilon} f$ can be reduced to that of the term $I I I_{\epsilon}$ in $(2.1)$.

Lemma 3. If $\epsilon_{0}$ is a given positive number, then there exists a large positive number $N=N(L)$ so that

$$
\left|\int_{\Gamma \backslash \Gamma_{z, N \epsilon}} T f(w) g_{z, \epsilon}(w) d w\right| \leq C M(T f)(z), \quad z \in \Gamma, \quad \epsilon_{0}<\epsilon,
$$

for a positive constant $C=C\left(\epsilon_{0}, L\right)$.

The small number $\epsilon_{0}$ will be chosen in the next section.

Proof. Recall that

$$
\begin{aligned}
g_{z, \epsilon}(w) & =T\left(K_{z, \epsilon}\right)(w)=-\frac{1}{\pi^{2}} \mathrm{p} \cdot \mathrm{v} \cdot \int_{\Gamma \backslash \Gamma_{z, \epsilon}} \frac{1}{(\zeta-w)(\zeta-z)} d \zeta \\
& =-\frac{1}{\pi^{2}} \frac{1}{w-z} \mathrm{p} \cdot \mathrm{v} \cdot \int_{\Gamma \backslash \Gamma_{z, \epsilon}}\left(\frac{1}{\zeta-w}-\frac{1}{\zeta-z}\right) d \zeta \\
& =-\frac{1}{\pi^{2}} \frac{1}{w-z} \text { p.v. } \int_{\Gamma \backslash \Gamma_{z, \epsilon}} \frac{1}{\zeta-w} d \zeta+\frac{1}{\pi^{2}} \frac{1}{w-z} \mathrm{p} \cdot \mathrm{v} \cdot \int_{\Gamma \backslash \Gamma_{z, \epsilon}} \frac{1}{\zeta-z} d \zeta \\
& =h(w)+k(w),
\end{aligned}
$$

where in the last identity we defined $h(w)$ and $k(w)$.

Applying the bilipschitz character of $\gamma$ we conclude that

$$
|k(w)| \leq \frac{1}{\pi^{2}} \frac{L^{2}}{N \epsilon_{0}^{2}} \operatorname{length}(\Gamma), \quad w \in \Gamma \backslash \Gamma_{z, N \epsilon}, \quad \epsilon_{0}<\epsilon .
$$

The estimate of $h(w)$ is a little trickier. We have

$$
h(w)=-\frac{1}{\pi^{2}} \frac{1}{w-z} \mathrm{p} \cdot \mathrm{v} \cdot \int_{\Gamma} \frac{1}{\zeta-w} d \zeta+\frac{1}{\pi^{2}} \frac{1}{w-z} \mathrm{p} \cdot \mathrm{v} \cdot \int_{\Gamma_{z, \epsilon}} \frac{1}{\zeta-w} d \zeta
$$


A simple application of Cauchy's Theorem gives that, if $\Gamma$ has a tangent at $w$,

$$
\text { p. v. } \int_{\Gamma} \frac{1}{\zeta-w} d \zeta=\pi i \text {. }
$$

As before, the bilipschitz character of $\gamma$ yields

$$
|w-z| \geq \frac{N \epsilon}{L}, \quad w \in \Gamma \backslash \Gamma_{z, N \epsilon}
$$

and

$$
|w-\zeta| \geq|w-z|-|z-\zeta| \geq \epsilon\left(\frac{N}{L}-L\right), \quad w \in \Gamma \backslash \Gamma_{z, N \epsilon}, \quad \zeta \in \Gamma_{z, \epsilon}
$$

Choose $N$ so that $N / L-L \geq 1$. Then

$$
|w-\zeta| \geq \epsilon, \quad w \in \Gamma \backslash \Gamma_{z, N \epsilon}, \quad \zeta \in \Gamma_{z, \epsilon} .
$$

Gathering all the previous estimates we finally get

$$
|h(w)| \leq \frac{1}{\pi} \frac{L}{N \epsilon_{0}}+\frac{1}{\pi^{2}} \frac{\text { length }(\Gamma)}{\epsilon_{0}}, \quad w \in \Gamma \backslash \Gamma_{z, N \epsilon}, \quad \epsilon_{0}<\epsilon .
$$

Hence (3.1) and (3.2) yield

$$
\left|g_{z, \epsilon}(w)\right| \leq C, \quad w \in \Gamma \backslash \Gamma_{z, N \epsilon}, \quad \epsilon_{0}<\epsilon,
$$

where $C=C\left(\epsilon_{0}, N, L\right.$, length $\left.(\Gamma)\right)$ is a constant depending on $\epsilon_{0}, N, L$ and length $(\Gamma)$.

Therefore

$$
\left|\int_{\Gamma \backslash \Gamma_{z, N \epsilon}} T f(w) g_{z, \epsilon}(w) d w\right| \leq C \int_{\Gamma}|T f(w)| d \mu(w) \leq C \operatorname{length}(\Gamma) M(T f)(z),
$$

which completes the proof of the lemma.

\section{The proof of the Theorem}

For $z \neq 0$ let $\operatorname{Arg}(z)$ denote the principal argument of $z$, so that $0 \leq \operatorname{Arg}(z)<2 \pi$.

Lemma 4. Given $\alpha>0$ there exists a positive number $\epsilon_{0}=\epsilon_{0}(L)$ with the following property. Assume that $0<\epsilon_{1} \leq \epsilon_{0}, \epsilon_{1} / 2<\epsilon \leq \epsilon_{1}$ and that for a fixed $x \in \mathbf{R}$ we have $\gamma(x)=0$. If $\gamma(x-\tau), \tau>0$, satisfies

$$
\frac{\epsilon_{1}}{2 L}<|\gamma(x-\tau)|<L \epsilon_{1}
$$

then, for some $\theta$ such that $\gamma(x-\tau)=|\gamma(x-\tau)| e^{i \theta}$, we have

$$
|\theta-(\operatorname{Arg}(\gamma(x+\epsilon))+\pi)|<\alpha .
$$

Proof. Consider the triangle with vertices $0, \gamma(x-\tau)$ and $\gamma(x+\epsilon)$ and side lengths $A=|\gamma(x-\tau)|, B=|\gamma(x+\epsilon)|$ and $C=|\gamma(x+\epsilon)-\gamma(x-\tau)|$. By the cosine Theorem

$$
C^{2}=A^{2}+B^{2}-2 A B \cos (\phi)
$$

where $\phi$ is the angle opposite to the side $C$. In other terms

$$
1+\cos (\phi)=\frac{(A+B-C)(A+B+C)}{2 A B} .
$$


By asymptotic conformality, given $\delta>0$ there exists $\eta_{0}>0$ such that $C=\mid \gamma(x+$ $\epsilon)-\gamma(x-\tau) \mid<\eta_{0}$ implies $A+B \leq(1+\delta) C$. The bilipschitz property of $\gamma(1.5)$ yields $\epsilon_{1} / 2 L^{2} \leq \tau \leq L^{2} \epsilon_{1}$. Hence

$$
1+\cos (\phi) \leq \delta L^{4} \frac{\left(\epsilon_{1}+\tau\right)^{2}}{\epsilon_{1} \tau} \leq 2 \delta L^{6}\left(1+L^{2}\right)^{2} .
$$

Taking $\theta=\operatorname{Arg}(\gamma(x+\epsilon))+\phi$ we see that $|\theta-(\operatorname{Arg}(\gamma(x+\epsilon))+\pi)|<\alpha$ provided $\delta$ is small enough. Since

$$
|\gamma(x+\epsilon)-\gamma(x-\tau)| \leq L(\epsilon+\tau) \leq \epsilon_{0} L\left(1+L^{2}\right)
$$

one has to choose $\epsilon_{0}$ so that $\epsilon_{0} L\left(1+L^{2}\right) \leq \eta_{0}$, which shows the correct dependence of $\epsilon_{0}$ and completes the proof of the Lemma.

Given a point $z \in \Gamma$ we want now to construct a special Jordan arc $\Delta_{z}$ connecting $z$ to $\infty$ in the complement of $\Gamma$. Assume, without loss of generality, that $z=0$. Take $x \in \mathbf{R}$ with $\gamma(x)=0$. Let $\epsilon_{0}$ be the number given in the preceding lemma and define, for $j=0,1,2, \ldots$, a polar rectangle by

$$
R_{j}=\left\{w=|w| e^{i \theta}: \frac{\epsilon_{0}}{2^{j+1} L}<|w|<\frac{\epsilon_{0} L}{2^{j}} \quad \text { and } \quad\left|\theta-\operatorname{Arg}\left(\gamma\left(x+\frac{\epsilon_{0}}{2^{j}}\right)\right)+\pi\right|<\alpha\right\} .
$$

Applying Lemma 4 with $\epsilon=\epsilon_{1}=\epsilon_{0} / 2^{j}$ we conclude that

$$
\{\gamma(x-\tau): 0<\tau\} \cap\left\{w: \frac{\epsilon_{0}}{2^{j+1} L}<|w|<\frac{\epsilon_{0} L}{2^{j}}\right\} \subset R_{j} .
$$

We need to introduce another polar rectangle

$$
S_{j}=R_{j} \cap\left\{w: \frac{\epsilon_{0} L}{2^{j+1}}<|w|\right\}, \quad j=0,1,2, \ldots
$$

We define inductively $\Delta_{z}=\Delta_{0}$ on $S_{j}$ by just requiring that the Jordan arc $\Delta_{0} \cap \overline{S_{j}}$ lies in the unbounded component of the complement of $\Gamma, \overline{S_{j}}$ being the closure of $S_{j}$. We then connect $\Delta_{0} \cap \overline{S_{0}}$ with $\infty$ by a Jordan arc in the complement of $\Gamma$, with the only precaution of not reentering the disc $D\left(0, \epsilon_{0}\right)$ once $\Delta_{0}$ has left it.

It is worth pointing out that the axis of two consecutive polar rectangles $R_{j}$ and $R_{j+1}$ make an angle less than $\alpha$. This follows by the defining property of $\epsilon_{0}$ (see the proof of Lemma 4).

\section{Lemma 5.}

$$
\log (\gamma(x-\epsilon))-\pi i=\log (-\gamma(x-\epsilon)), \quad x \in \mathbf{R}, \quad 0<\epsilon \leq \epsilon_{0} .
$$

Proof. We know that

$$
\log (\gamma(x-\epsilon))-\pi i=\log (-\gamma(x-\epsilon))+2 \pi m i
$$

for some integer $m$. Our goal is to compute the difference

$$
\log (\gamma(x-\epsilon))-\log (-\gamma(x-\epsilon))
$$

by the integral

$$
\int_{\varsigma} \frac{1}{z} d z
$$

where $\varsigma$ is an appropriately chosen Jordan arc connecting $-\gamma(x-\epsilon)$ to $\gamma(x-\epsilon)$ in the complement of $\Delta_{0}$. 


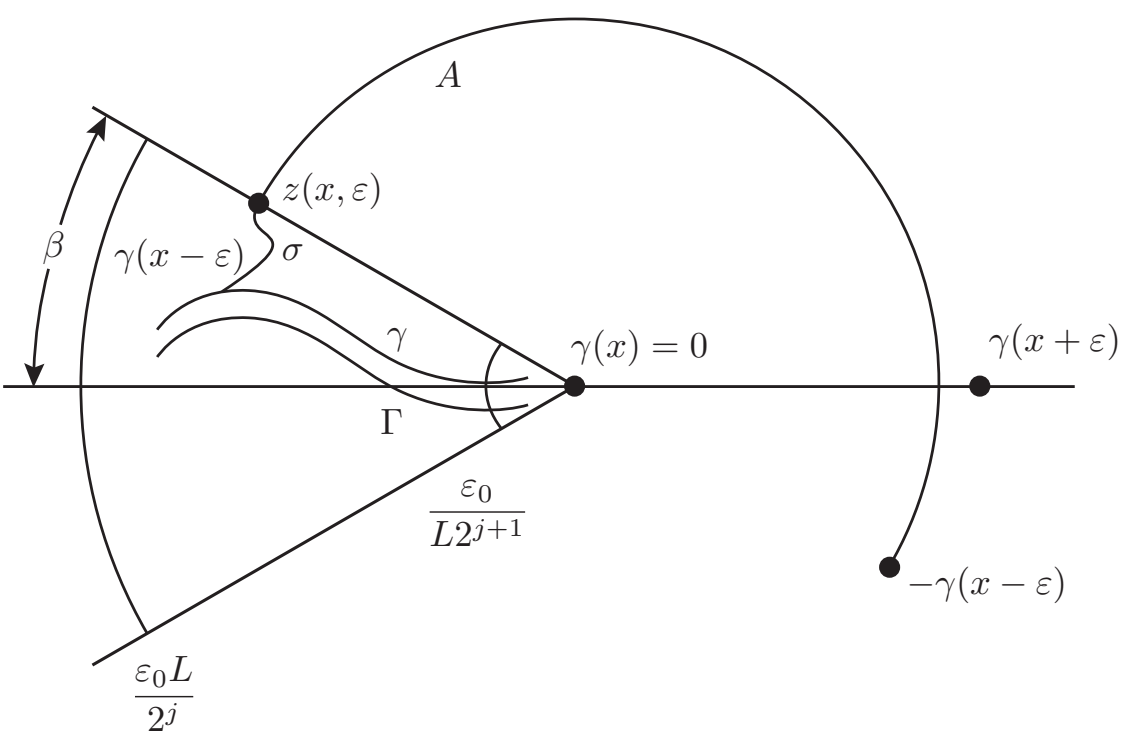

Figure 1. The curve $\varsigma$.

Assume that $\epsilon_{0} / 2^{j+1}<\epsilon \leq \epsilon_{0} / 2^{j}$, for some non-negative integer $j$. Define $N$ as the smallest integer satisfying

$$
\frac{L \epsilon_{0}}{2^{j+N}} \leq \frac{\epsilon_{0}}{L 2^{j+1}}
$$

This is equivalent to $L^{2} \leq 2^{N-1}$ and so $N$ depends only on $L$. Hence $R_{k} \subset$ $D\left(0, \epsilon_{0} / L 2^{j+1}\right), k \geq j+N$, and, in particular, $R_{k}, k \geq j+N$, does not intersect the circumference $\partial D(0,|\gamma(x-\epsilon)|)$.

The angle between the axis of the polar rectangle $R_{j+l}$ and that of $R_{j}$ is not greater than $l \alpha \leq N \alpha, l=1,2, \ldots, N-1$. Set $\beta=N \alpha$, so that $\beta$ can be as small as desired by taking $\alpha=\alpha(L)$ appropriately. We conclude that

$$
R_{j+l} \subset\left\{w: w=|w| e^{i \theta} \text { with }|\theta-\operatorname{Arg}(\gamma(x+\epsilon)+\pi)|<\beta\right\}, \quad l=1,2, \ldots, N-1 .
$$

We are now ready to define the Jordan arc $\varsigma$. Let $z(x, \epsilon)$ be the point at the intersection of the circumference $\partial D(0,|\gamma(x-\epsilon)|)$ and the ray

$$
\left\{w: w=|w| e^{i \theta} \text { with } \theta=\operatorname{Arg}(\gamma(x+\epsilon)+\pi)-\beta\right\} .
$$

Let $A$ stand for the arc in $\partial D(0,|\gamma(x-\epsilon)|)$ having $-\gamma(x-\epsilon)$ as initial point and $z(x, \epsilon)$ as end point (counterclockwise oriented).

There exists a rectifiable Jordan arc $\sigma$ joining the points $z(x, \epsilon)$ and $\gamma(x-\epsilon)$ in the bounded component of the complement of $\Gamma$ with the property that

$$
\operatorname{length}(\sigma) \leq C|z(x, \epsilon)-\gamma(x-\epsilon)| .
$$

This can be seen readily as follows. Set $\tilde{\gamma}\left(e^{i x}\right)=\gamma(x), x \in \mathbf{R}$. Then $\tilde{\gamma}$ is a bilipschitz homeomorphism between $\mathbf{T}$ and $\Gamma$ and thus can be extended to a global bilipschitz homeomorphism of the plane onto itself (see [7, 8]). The existence of the arc $\sigma$ is then easily proved by transferring the question via the extended bilipschitz homeomorphism.

Define $\varsigma=A \cup \sigma$, oriented as already specified. Note that $\varsigma$ lies in the complement of $\Delta_{0}$, by the previous discussion, in particular, the definition of $N$ and $\beta$. Therefore

$$
\log (\gamma(x-\epsilon))-\log (-\gamma(x-\epsilon))=\int_{\varsigma} \frac{1}{z} d z
$$


On one hand we have

$$
\int_{A} \frac{1}{z} d z=\pi i+O(\beta)
$$

and on the other hand

$$
\left|\int_{\sigma} \frac{1}{z} d z\right| \leq \frac{C|z(x, \epsilon)-\gamma(x-\epsilon)|}{|\gamma(x-\epsilon)|} \leq C \beta=O(\beta) .
$$

If $\beta$ is small enough so that $O(\beta)<\pi$, then, by (4.1), we get that $m=0$, and the lemma is proved.

We need a final lemma, which concludes the proof of the Theorem.

Lemma 6. Let $\Gamma$ be an asymptotically conformal chord-arc curve and let $\gamma$ be a bilipschitz parametrization of $\Gamma$ (in the sense of (1.5)). Then there exists a constant $C>1$ and a positive number $\epsilon_{0}$ such that

$$
\begin{aligned}
C^{-1} \frac{|\gamma(x+\epsilon)+\gamma(x-\epsilon)-2 \gamma(x)|}{\epsilon} & \leq|F(x, \epsilon)| \\
& \leq C \frac{|\gamma(x+\epsilon)+\gamma(x-\epsilon)-2 \gamma(x)|}{\epsilon},
\end{aligned}
$$

for $x \in \mathbf{R}$ and $0<\epsilon<\epsilon_{0}$.

Proof. Without loss of generality assume that $\gamma(x)=0$. Let $\epsilon_{0}$ be the small number provided by Lemma 4 . By the construction of the arc $\Delta_{0}$ described in the proof of Lemma 4 we have that the segment joining $-\gamma(x-\epsilon)$ and $\gamma(x+\epsilon)$ lies in the complement of $\Delta_{0}$. We have, by Lemma 5 ,

$$
\begin{aligned}
F(x, \epsilon) & =\log (\gamma(x+\epsilon))-\log (\gamma(x-\epsilon))+\pi i \\
& =\log (\gamma(x+\epsilon))-\log (-\gamma(x-\epsilon))
\end{aligned}
$$

and so

$$
\begin{aligned}
F(x, \epsilon) & =\int_{0}^{1} \frac{d}{d t} \log (-\gamma(x-\epsilon)+t(\gamma(x+\epsilon)+\gamma(x-\epsilon))) d t \\
& =\int_{0}^{1} \frac{\gamma(x+\epsilon)+\gamma(x-\epsilon)}{-\gamma(x-\epsilon)+t(\gamma(x+\epsilon)+\gamma(x-\epsilon))} d t .
\end{aligned}
$$

Set, to simplify notation, $a=-\gamma(x-\epsilon), b=\gamma(x+\epsilon)$ and let $\theta$ denote the angle between $a$ and $b$. By Lemma 4 we know that $\theta$ is as small as we wish. In particular we can assume that $\cos (\theta) \geq 1 / 2$. Thus, using the cosine Theorem,

$$
\begin{aligned}
|a+t(b-a)|^{2} & =(1-t)^{2}|a|^{2}+t^{2}|b|^{2}+2(1-t) t|a||b| \cos (\theta) \\
& \geq \frac{1}{2}((1-t)|a|+t|b|)^{2} \geq \frac{\epsilon^{2}}{2 L^{2}},
\end{aligned}
$$

and

$$
|F(x, \epsilon)| \leq \frac{\sqrt{2} L}{\epsilon}|\gamma(x+\epsilon)+\gamma(x-\epsilon)|
$$

which is the upper estimate in (4.2).

For the lower estimate we set $z_{t}=-\gamma(x-\epsilon)+t(\gamma(x+\epsilon)+\gamma(x-\epsilon))$. Since $\operatorname{Re}\left(z_{t}\right) \geq\left|z_{t}\right| / 2$ and $\left|z_{t}\right| \leq 2 L \epsilon$

$$
\left|\int_{0}^{1} \frac{1}{z_{t}} d t\right| \geq \operatorname{Re} \int_{0}^{1} \frac{1}{z_{t}} d t=\int_{0}^{1} \frac{\operatorname{Re}\left(z_{t}\right)}{\left|z_{t}\right|^{2}} d t \geq \int_{0}^{1} \frac{1}{2\left|z_{t}\right|} d t \geq \frac{1}{4 L \epsilon}
$$


To complete the proof of the Theorem one only needs to combine Lemmas 2, 3 and 6.

Remark. Let $a=\gamma(x)-\gamma(x-\epsilon), b=\gamma(x+\epsilon)-\gamma(x)$ and let $\alpha(x, \epsilon)$ be the angle spanned by $a$ and $b$. For a bilipschitz parametrization $\gamma$ such that

$$
c|x-y| \leq|\gamma(x)-\gamma(y)| \leq C|x-y|, \quad x, y \in \mathbf{R}, \quad|x-y| \leq \frac{T}{2},
$$

we have the estimate

$$
|\gamma(x+\epsilon)+\gamma(x-\epsilon)-2 \gamma(x)|^{2} \leq 2 C^{2} \epsilon^{2}-2 c^{2} \epsilon^{2} \cos \alpha(x, \epsilon)
$$

So, in the general case, we can guarantee just a linear decay of the second finite difference $|\gamma(x+\epsilon)+\gamma(x-\epsilon)-2 \gamma(x)|$ and the logarithmic condition (1.7) gives informations about the local behavior of the best constants $c$ and $C$ around $x$ and about the decay of $\alpha(x, \epsilon)$ for $\epsilon$ small. This remark will be useful in the next section.

\section{An example}

In this section we provide an example of curve $\gamma$ which is not $C^{1}$ but for which the improved Cotlar's inequality (1.6) holds. The curve will be constructed in a recursive way and will be parametrized by arc-length. Without loss of generality, we will focus on defining a curve which is not closed. Indeed, possibly by connecting the ends of this curve in a smooth way, we can reduce to the same environment of the previous sections. The idea in the construction of the example is that the curve should resemble a suitable spiraling sequence of smoothened corners of decreasing aperture.

Let $0<\alpha<\pi / 2$. Let $F_{\alpha}:[0,1] \rightarrow \mathbf{R}$ be the function with support in $[1 / 4,3 / 4]$ which is linear in $[1 / 4,1 / 2]$ and $[1 / 2,3 / 4]$ with slope $\tan \alpha$ in $[1 / 4,1 / 2]$ and $-\tan \alpha$ in $[1 / 2,3 / 4]$. In other words

$$
F_{\alpha}(t):=\max \left\{0,\left(\frac{1}{4}-\left|t-\frac{1}{2}\right|\right) \tan \alpha\right\} .
$$

Let $\xi>0$. For $t \in \mathbf{R}$ we define the function

$$
\eta_{\xi}(t):=\eta\left(\frac{t}{\xi}\right) \frac{1}{\xi}
$$

where $\eta$ is a smooth, even and positive function such that $\operatorname{supp} \eta \subset[-1,1]$ and $\int \eta(t) d t=1$. For $0<\xi<1 / 100$ we define the regularized function

$$
\lambda_{\alpha}:=F_{\alpha} * \eta_{\xi} .
$$

We will call the curve $\Lambda_{\alpha}:=\left(t, \lambda_{\alpha}(t)\right)_{t \in[0,1]} \alpha$-patch.

An $\alpha$-patch has the following properties:

- $\Lambda_{\alpha}$ is the graph of a function $\lambda_{\alpha}:[0,1] \rightarrow \mathbf{R}$ which is symmetric around $1 / 2$.

- if we denote by $[a, b]$ the segment joining the points $a, b \in \mathbf{R}^{2}$, then $\Lambda_{\alpha}$ contains the segments $I_{\alpha}:=[(0,0),(1 / 4-\xi, 0)], I I_{\alpha}:=[(1 / 4+\xi, \xi \tan \alpha),(1 / 2-$ $\xi,(1 / 4-\xi) \tan \alpha)], I I I_{\alpha}:=[(1 / 2+\xi,(1 / 4-\xi) \tan \alpha),(3 / 4-\xi, \xi \tan \alpha)]$ and $I V_{\alpha}:=[(3 / 4+\xi, 0),(1,0)]$. We denote by $C_{\alpha}^{i}, i=1,2,3$ the remaining three non-affine parts of the graph. Precisely, $C_{\alpha}^{1}$ joins the segments $I_{\alpha}$ and $I I_{\alpha}$, $C_{\alpha}^{2}$ the segments $I I_{\alpha}$ and $I I I_{\alpha}$ and $C_{\alpha}^{3}$ the segments $I I I_{\alpha}$ and $I V_{\alpha}$.

- the function $\lambda_{\alpha}$ is convex on the intervals below $C_{\alpha}^{1}$ and $C_{\alpha}^{3}$ and concave on the interval below $C_{\alpha}^{2}$.

The idea is that the $\alpha$-patch is a smoothened corner, as shown in Figure 2. 


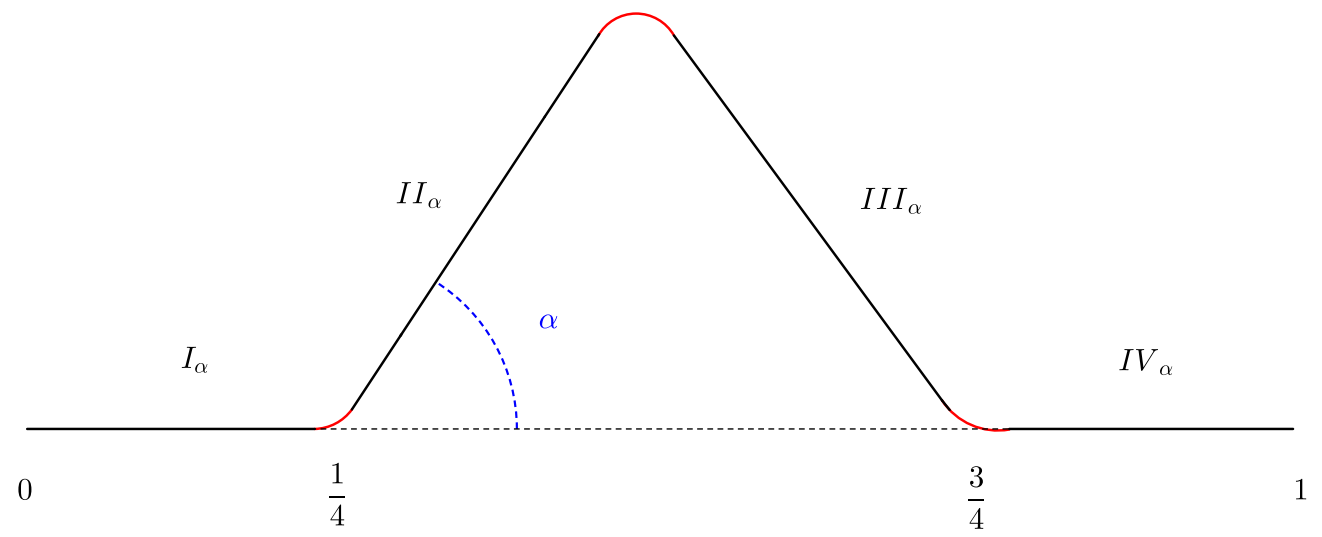

Figure 2. An $\alpha$-patch.

Remark 1. Let us denote by $\tau(\alpha)$ the difference between the length of the (nonsmoothened) graph of $F_{\alpha}$ and the length of $\Lambda_{\alpha}$. For what follows, we need to estimate its behavior for small values of $\alpha$. It suffices to observe that

$$
\begin{aligned}
\tau(\alpha) & :=\operatorname{length}\left(F_{\alpha}\right)-\operatorname{length}\left(\Lambda_{\alpha}\right) \\
& =\int_{0}^{1}\left(\sqrt{1+\left|f_{\alpha}^{\prime} * \eta_{\xi}\right|^{2}(t)}\right)-\left(\sqrt{1+\left|f_{\alpha}^{\prime}\right|^{2}(t)}\right) d t \\
& =\int_{0}^{1} \frac{\left|f_{\alpha}^{\prime} * \eta_{\xi}\right|^{2}(t)-\left|f_{\alpha}^{\prime}\right|^{2}(t)}{\left(\sqrt{1+\left|f_{\alpha}^{\prime} * \eta_{\xi}\right|^{2}(t)}\right)+\left(\sqrt{1+\left|f_{\alpha}^{\prime}\right|^{2}(t)}\right)} d t \leq 2\left\|f_{\alpha}^{\prime}\right\|_{\infty}=2 \tan \alpha .
\end{aligned}
$$

Definition of the curve $\boldsymbol{\Gamma}$. Let $\alpha_{j}:=1 / j$ for $j=1,2, \ldots$ positive integer. For the sake of notational convenience we replace the subscript $\alpha_{j}$ by $j$; for instance, we write $\Lambda_{j}$ for $\Lambda_{\alpha_{j}}, I_{j}$ for $I_{\alpha_{j}}, \ldots, I V_{j}$ for $I V_{\alpha_{j}}$ and $C_{j}^{i}$ for $C_{\alpha_{j}}^{i}$. Moreover, $\tau_{j}:=\tau\left(\alpha_{j}\right)$. Now we can define $\Gamma$ according to the following recursive steps:

- $\Gamma_{1}:=\Lambda_{1}$.

- We would like to glue on $I I_{1}$ an appropriate rescaled, translated and rotated copy $\tilde{\Lambda}_{2}$ of $\Lambda_{2}$. The angle of rotation is $\alpha_{1}$. The scaling factor and the translation are chosen so that the origin of $\tilde{\Lambda}_{2}$ is $(1 / 4,0)$ and the end is $(1 / 2,(\tan \alpha) / 4)$. Denote by $\widetilde{I I}_{2}$ the image of $I I_{2}$ via the same affinity which maps $\Lambda_{2}$ to $\tilde{\Lambda}_{2}$; let us use the tilde to denote the images of the other parts of the patch via the same map, too. Delete the segment $I I_{1}$ from $\Lambda_{1}$ and add $\tilde{\Lambda}_{2}$. Now the endings of $\tilde{\Lambda}_{2}$ should be deleted in order to make a connection with $\Lambda_{1}$. The precise expression for the second step curve is

$$
\Gamma_{2}:=\left(\left(\Lambda_{1} \backslash I I_{1}\right) \cup \tilde{\Lambda}_{2}\right) \backslash\left(\left(\tilde{I}_{2} \cup \widetilde{I V}_{2}\right) \backslash I I_{1}\right) .
$$

- given $\Gamma_{n}$, which is a "gluing" of affine copies $\tilde{\Lambda}_{j}$ of $\Lambda_{j}$ for $j \in\{1, \ldots, n\}$, where $\widetilde{I I}_{n}$ is the image of $I I_{j}$ under the same affinity which maps $\Lambda_{j}$ to $\tilde{\Lambda}_{j}$, we define

$$
\Gamma_{n+1}:=\left(\left(\tilde{\Lambda}_{n} \backslash \widetilde{I I}_{n}\right) \cup \tilde{\Lambda}_{n+1}\right) \backslash\left(\left(\widetilde{I}_{n+1} \cup \widetilde{I V}_{n+1}\right) \backslash \widetilde{I I}_{n}\right),
$$

where $\tilde{\Lambda}_{n+1}$ is an re-scaled copy of $\Lambda_{n+1}$ rotated by an angle $\sum_{j=1}^{n+1} \alpha_{j}$ whose vertices coincide with the images of $(1 / 4,0)$ and $(1 / 2, \tan \alpha / 4)$ via the transformation of the plain that sends $\Lambda_{n}$ to $\widetilde{\Lambda}_{n}$.

Then, $\left\{\Gamma_{n}\right\}_{n}$ converges in the Hausdorff distance (a similar case is presented, for example, in [1]) and we can simply define $\Gamma:=\lim _{n} \Gamma_{n}$. 


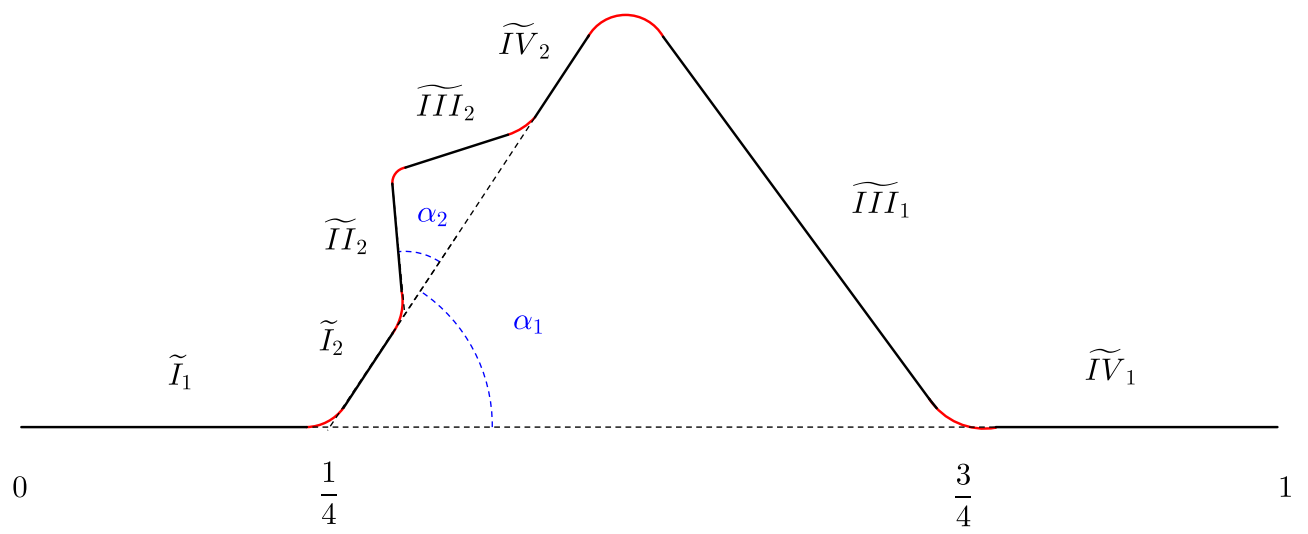

Figure 3. The second step in the construction of the curve $\Gamma$.

Let us now state an estimate that we will use in what follows.

Lemma 7. Given $0<\alpha<\pi / 2$ and $z_{1}, z_{2} \in \Lambda_{\alpha}$, we have

$$
l\left(z_{1}, z_{2}\right) \leq \frac{\left|z_{1}-z_{2}\right|}{\cos \alpha}
$$

where $l\left(z_{1}, z_{2}\right)$ denotes the length of the arc of $\Lambda_{\alpha}$ joining $z_{1}$ and $z_{2}$.

Proof. Let $t_{1}:=\lambda_{\alpha}^{-1}\left(z_{1}\right)$ and $t_{2}:=\lambda_{\alpha}^{-1}\left(z_{2}\right)$. We have $\left|t_{1}-t_{2}\right| \leq\left|z_{1}-z_{2}\right|$. Moreover, because of the way we constructed $\Lambda_{\alpha}$, we have that $\left|\lambda_{\alpha}^{\prime}(t)\right| \leq \tan \alpha$ for every $t \in[0,1]$. Collecting all these observations,

$$
\begin{aligned}
l\left(z_{1}, z_{2}\right) & =\int_{t_{1}}^{t_{2}} \sqrt{1+\left|\lambda_{\alpha}^{\prime}(t)\right|^{2}} d t \leq \int_{t_{1}}^{t_{2}} \sqrt{1+|\tan \alpha|^{2}} d t \\
& =\left|t_{2}-t_{1}\right| \sqrt{1+|\tan \alpha|^{2}}=\frac{\left|t_{2}-t_{1}\right|}{\cos \alpha} \leq \frac{\left|z_{2}-z_{1}\right|}{\cos \alpha} .
\end{aligned}
$$

Remark 2. Notice that the inequality (5.2) keeps holding for a scaling of $\Lambda_{\alpha}$, in particular for the $\tilde{\Lambda}_{j}, j \in \mathbf{N}$.

Let us define $L_{1}=1 / 2$ and, for $n>1$,

$$
L_{n}:=2^{-2 n+1}\left(\prod_{j=1}^{n-1} \cos \alpha_{j}\right)^{-1}
$$

which is half of the diameter of the rescaled patch $\tilde{\Lambda}_{n}$ in the construction of the curve $\Gamma$. Indeed, some trigonometry gives

$$
L_{1}=\frac{1}{2}, L_{2}=\frac{1}{2}\left(\frac{1}{2} L_{1} \frac{1}{\cos \alpha_{1}}\right), L_{3}=\frac{1}{2}\left(\frac{1}{2} L_{2} \frac{1}{\cos \alpha_{2}}\right), \cdots, L_{n}=\frac{1}{2}\left(\frac{1}{2} L_{n-1} \frac{1}{\cos \alpha_{n-1}}\right) .
$$

Observe that the definition of $L_{n}$ does not depend on $\alpha_{n}$ because the scaling of $\tilde{\Lambda}_{n}$ is determined just by the previous $(n-1)$ angles. We will use $L_{n}$ as a quantifier of the scale.

Lemma 8. For every $\delta>0$ there exists $k \in \mathbf{N}$ big enough such that for $z_{1}, z_{2} \in$ $\Gamma \cap\left(\bigcup_{j=k}^{\infty} \tilde{\Lambda}_{j}\right)$ we have

$$
l\left(z_{1}, z_{2}\right) \leq(1+\delta)\left|z_{1}-z_{2}\right|
$$


Proof. Let us start with some geometrical observation. Let $k \in \mathbf{N}$ and $\zeta_{1}, \zeta_{2} \in \Gamma$. Suppose, moreover, that $\zeta_{1} \in \tilde{I}_{k}$ and $\zeta_{2} \in \widetilde{I V}_{k}$. It is useful to define

$$
R_{k}:=l\left(\zeta_{1}, \zeta_{2}\right)-\left|\zeta_{1}-\zeta_{2}\right|
$$

Observe that the definition of $R_{k}$ does not depend on the choice of $\zeta_{1}$ and $\zeta_{2}$ in the respective segments. In particular, by the construction of the curve $\Gamma$ and by the definition of the error term $\tau_{j}$ in (5.1), it is not difficult to check that we have

$$
R_{k}=\left(3 \sum_{j=k+1}^{\infty} L_{j}-L_{k}\right)-\sum_{j=k+1}^{\infty} 2 L_{j} \tau_{j} .
$$

The term between parentheses in the right hand side is the length of the gluing of the 'non-regularized' $\alpha$-patches in the construction and the second sum is an error term due to the smoothing in the definition of $\alpha$-patch.

Because of how we chose $L_{j}$ and $\tau_{j}$, the quantity $R_{k}$ represents the error we make in estimating the length of the arch of the curve between $\zeta_{1} \in \tilde{I}_{k}$ and $\zeta_{2} \in \widetilde{I V}_{k}$ compared to $\left|\zeta_{1}-\zeta_{2}\right|$. The presence of factor $2 L_{j}$ in the last sum in the right hand side of (5.4) is due to the fact that the diameter of $\tilde{\Lambda}_{j}$ is equal to $2 L_{j}$ and, thus, the error term $\tau_{j}$ has to be rescaled by that value. It turns out that

$$
\frac{R_{k}}{L_{k}} \rightarrow 0 \quad \text { as } k \rightarrow \infty
$$

which justifies the interpretation of $R_{k}$ as an error term. Indeed, recalling that $\cos \alpha_{l} \geq \cos \alpha_{k}$ for $l \geq k$, we have

$$
\begin{aligned}
\frac{3}{L_{k}} \sum_{j=k+1}^{\infty} L_{j} & =\sum_{j=k+1}^{\infty} \frac{3}{4^{j-k}}\left(\prod_{l=k}^{j-1} \cos \alpha_{l}\right)^{-1} \\
& \leq 3 \sum_{j=k+1}^{\infty}\left(\frac{1}{4 \cos \alpha_{k}}\right)^{j-k}=\frac{3}{4 \cos \alpha_{k}-1}
\end{aligned}
$$

and the last term tends to 1 as $k \rightarrow \infty$. Moreover, using (5.1) and since $L_{j} \leq 2^{k-j} L_{k}$ for $j>k$, we have that

$$
\frac{1}{L_{k}} \sum_{j=k+1}^{\infty} 2 L_{j} \tau_{j} \lesssim \tau_{k+1} \sum_{j=k+1}^{\infty} 2^{k-j} \rightarrow 0 \quad \text { as } k \rightarrow \infty,
$$

so that (5.5) follows.

Let us combine this observation with (5.2) to prove (5.3). Let $z_{1}, z_{2} \in \Gamma$. Observe that each point of $\Gamma$ belongs to $\widetilde{\Lambda}_{j}$ for at most two different $j$. Let $k_{1}$ be the maximum index such that $z_{1} \in \tilde{\Lambda}_{k_{1}}$ and let $k_{2}$ be the maximum index such that $z_{2} \in \tilde{\Lambda}_{k_{2}}$. The rest of the proof works with minor changes if we take the minimum instead of the maximum in the definitions of $k_{1}$ and $k_{2}$. The use of this indices helps to make the calculations more systematic.

Without loss of generality, suppose $k_{1} \leq k_{2}$. If $k_{1}=k_{2}$, the points belong to the image of the same patch. We have two possible scenarios depending on the relative position of these points. The definition of $R_{k}$ and the estimate (5.2) allow us to write

$$
l\left(z_{1}, z_{2}\right) \leq \frac{\left|z_{1}-z_{2}\right|}{\cos \alpha_{k_{1}}},
$$


if the point are at a distance $\left|z_{1}-z_{2}\right| \leq L_{k_{1}+1}$. For $\left|z_{1}-z_{2}\right| \geq L_{k_{1}+1} / 4$, we have to consider the additional error term $R_{k_{1}+1}$, which comes from the 'spiraling' part of the curve. In particular

$$
l\left(z_{1}, z_{2}\right) \leq \frac{\left|z_{1}-z_{2}\right|}{\cos \alpha_{k_{1}}}+R_{k_{1}+1} \leq \frac{\left|z_{1}-z_{2}\right|}{\cos \alpha_{k_{1}}}+\frac{R_{k_{1}+1}}{4 L_{k_{1}+1}}\left|z_{1}-z_{2}\right|,
$$

so that, invoking (5.5), the lemma is proven in the case $k_{1}=k_{2}$.

Let us consider the other case, $k_{1}<k_{2}$. If $z_{2} \in \widetilde{\Lambda}_{k_{1}}$, (5.6) easily applies because the two points belong to the image of the same patch. So we can suppose $z_{2} \notin \widetilde{\Lambda}_{k_{1}}$. In this case

$$
\left|z_{1}-z_{2}\right| \geq \frac{L_{k_{1}+1}}{4}
$$

Let $z_{2}^{\prime} \in \widetilde{I I}_{k_{1}}$ be the orthogonal projection of $z_{2}$ on the segment $\widetilde{I I}_{k_{1}}$. The idea now is, by means of projections, to reduce to the case in which the points belong to the image of the same patch. For this purpose it is also useful to use the length of the arcs of the $m$-th step curve $\Gamma_{m}$ that we used to define $\Gamma$. By the triangular inequality and denoting by

(5.8) $h_{k_{1}+1}:=\min \left\{h: \widetilde{\Lambda}_{k_{1}+1} \subset[0, h] n_{V}+V\right.$ for some affine line $V$ with normal $\left.n_{V}\right\}$ the width of $\tilde{\Lambda}_{k_{1}+1}$, we have

$$
\left|z_{1}-z_{2}^{\prime}\right| \leq\left|z_{1}-z_{2}\right|+h_{k_{1}+1}
$$

Let us remark that, by construction of $\Gamma$,

$$
\frac{h_{k_{1}+1}}{L_{k_{1}+1}} \rightarrow 0 \quad \text { as } k \rightarrow \infty
$$

Given $m \in \mathbf{N}$ and $u, v \in \Gamma_{m}$, it is useful to denote by $l_{m}(u, v)$ the length of the arc of $\Gamma_{m}$ joining $u$ and $v$. Now we want to prove that

$$
l\left(z_{1}, z_{2}\right) \leq l_{k_{1}}\left(z_{1}, z_{2}^{\prime}\right)+R_{k_{1}+1} .
$$

Let us just consider the case $z_{1} \in \tilde{I}_{k_{1}}$, since the other cases are analogous. If $z_{k_{2}} \in$ $\tilde{I}_{k_{1}+1}$ or $z_{k_{2}} \in \widetilde{I V}_{k_{1}+1},(5.11)$ holds trivially because $z_{2}=z_{2}^{\prime}$. Otherwise, let $\zeta$ be a point on $\widetilde{I V}_{k_{1}+1}$ and let us consider the quantities $l\left(z_{2}, \zeta\right)$ and $\left|z_{2}^{\prime}-\zeta\right|$. Observe that the consideration below does not depend on the auxiliary point $\zeta$ of $\widetilde{I V}_{k_{1}+1}$ we choose. Clearly $l\left(z_{2}, \zeta\right) \geq\left|z_{2}^{\prime}-\zeta\right|$ and, because of the definition of $R_{k_{1}+1}$, the equality

$$
l\left(z_{1}, z_{2}\right)+l\left(z_{2}, \zeta\right)=R_{k_{1}+1}+l_{k_{1}}\left(z_{1}, z_{2}^{\prime}\right)+\left|z_{2}^{\prime}-\zeta\right|,
$$

holds. So

$$
l\left(z_{1}, z_{2}\right)=l_{k_{1}}\left(z_{1}, z_{2}^{\prime}\right)+R_{k_{1}+1}+\left(\left|z_{2}^{\prime}-\zeta\right|-l\left(z_{2}, \zeta\right)\right) \leq l_{k_{1}}\left(z_{1}, z_{2}^{\prime}\right)+R_{k_{1}+1} .
$$

The proof of the lemma is now over: indeed using (5.2), (5.5), (5.7), (5.9) and (5.10) we get

$$
\begin{aligned}
\frac{l\left(z_{1}, z_{2}\right)}{\left|z_{1}-z_{2}\right|} & \leq \frac{l_{k_{1}}\left(z_{1}, z_{2}^{\prime}\right)}{\left|z_{1}-z_{2}\right|}+\frac{R_{k_{1}+1}}{\left|z_{1}-z_{2}\right|} \leq \frac{\left|z_{1}-z_{2}^{\prime}\right|}{\left|z_{1}-z_{2}\right| \cos \alpha_{k_{1}}}+\frac{R_{k_{1}+1}}{\left|z_{1}-z_{2}\right|} \\
& \leq \frac{1}{\cos \alpha_{k_{1}}}+\frac{4 h_{k_{1}+1}}{\cos \alpha_{k_{1}} L_{k_{1}+1}}+\frac{4 R_{k_{1}+1}}{L_{k_{1}+1}} \rightarrow 1 \quad \text { as } k_{1} \rightarrow \infty
\end{aligned}
$$


A rectifiable curve $\Gamma$ is said asymptotically smooth if, denoting by $l\left(w_{1}, w_{2}\right)$ the length of the shortest arc of $\Gamma$ between $w_{1}, w_{2} \in \Gamma$,

$$
\frac{l\left(w_{1}, w_{2}\right)}{\left|w_{1}-w_{2}\right|} \rightarrow 1 \quad \text { as } \quad\left|w_{1}-w_{2}\right| \rightarrow 0, \quad w_{1}, w_{2} \in \Gamma .
$$

As shown in [6], an asymptotically smooth curve is also asymptotically conformal.

Proposition 1. $\Gamma$ is asymptotically smooth but not $C^{1}$.

Proof. Let $\tilde{z}_{j}^{\prime} \in \Gamma$ be the image of the point $z_{\alpha_{j}}^{\prime}$ via the map which sends $\Lambda_{j}$ to $\widetilde{\Lambda}_{j}$. We have that the curve $\Gamma$ is not $C^{1}$ at the point $z_{0}:=\lim _{j} z_{j}$, where $z_{j}$ is an arbitrary point of $\tilde{\Lambda}_{j}$. Indeed, by our choice of the angles in the construction, $\sum_{j} \alpha_{j}=+\infty$ and the curve spirals close to $z_{0}$.

Let us now turn prove that the curve is asymptotically smooth. Notice that we may write $\Gamma=\Gamma_{1} \cup \Gamma_{2} \cup\left\{z_{0}\right\}$, where $\Gamma_{1}$ and $\Gamma_{2}$ are smooth curves. Then, for every couple of points $\left\{z_{1}, z_{2}\right\}$ in one of those two smooth components we can exploit the smoothness to state that for every $\delta$ there exists $\bar{\epsilon}$ such that for $\epsilon<\bar{\epsilon}$ and $\left|z_{1}-z_{2}\right|=\epsilon$ we have

$$
l\left(z_{1}, z_{2}\right) \leq(1+\delta) \epsilon
$$

This, together with the result of Lemma 8 concludes the proof.

Let us consider the arc-length parametrization $\gamma$ of $\Gamma$. Being $\Gamma$ asymptotically smooth, $\gamma$ is bilipschitz. In particular,

$$
\frac{1}{C}|x-y| \leq|\gamma(x)-\gamma(y)| \leq|x-y|
$$

for a constant $C>1$ and $x, y \in[0, L(\Gamma)]$. As in Remark 4 we denote by $\alpha(x, \epsilon)$ the angle between the vectors $\gamma(x)-\gamma(x-\epsilon)$ and $\gamma(x+\epsilon)-\gamma(x)$. Because of the geometrical considerations in Remark 4, we have that

$$
|\gamma(x+\epsilon)+\gamma(x-\epsilon)-2 \gamma(x)|^{2} \leq \epsilon^{2}\left(2-\frac{2}{C^{2}} \cos \alpha(x, \epsilon)\right)
$$

for $\epsilon>0$ and $x \in[0, L(\Gamma)]$. Now we want to prove the estimate

$$
|\gamma(x+\epsilon)+\gamma(x-\epsilon)-2 \gamma(x)| \lesssim \frac{\epsilon}{|\log \epsilon|}
$$

Being $\Gamma$ smooth off the point $z_{0}$ and arguing as in [2], the logarithmic condition (1.4) and the estimate (1.6) are satisfied off that point. Hence it suffices to prove (1.6) for $\gamma(x) \in \bigcup_{k>k_{0}} \tilde{\Lambda}_{k} \cap \Gamma$ and $k_{0}$ big enough. To do that, we will study the behavior of the angle $\alpha(x, \epsilon)$ and of the local value of the bilipschitz constant of $\gamma$ close to the point $z_{0}$.

Being the curve asymptotically smooth, as a corollary of Lemma 4 we know that $\alpha(x, \epsilon) \rightarrow 0$ for $\epsilon$ small. Then, the second factor in the right hand side of (5.12) behaves as

$$
2-\frac{2}{C^{2}} \cos \alpha(x, \epsilon)=\left[2-\frac{2}{C^{2}}\right]+\frac{2}{C^{2}} \alpha(x, \epsilon)^{2}+O\left(\alpha(x, \epsilon)^{4}\right)
$$

for $\epsilon \rightarrow 0$.

Let $x_{0}:=\gamma^{-1}\left(z_{0}\right)$. For $\epsilon>0$, we denote by $C_{\epsilon}$ the smallest constant such that

$$
\frac{1}{C_{\epsilon}}|x-y| \leq|\gamma(x)-\gamma(y)| \leq|x-y|
$$


holds for $x, y \in\left[x_{0}-\epsilon, x_{0}+\epsilon\right]$, i.e. the local value of the lower bilipschitz constant close to $x_{0}$.

Using this notation, to our purposes it suffices to prove that

$$
|\alpha(x, \epsilon)| \lesssim|\log \epsilon|^{-1}
$$

and

$$
\left[1-\frac{1}{C_{\epsilon}}\right] \lesssim|\log \epsilon|^{-1}
$$

for $\epsilon$ small and $\gamma(x)$ close enough to $z_{0}$.

The following two lemmas respectively prove the estimate for the angle and the estimate for $C_{\epsilon}$.

Lemma 9. For every $\epsilon_{0}$ there exists an integer $k_{0}$ such that

$$
|\alpha(x, \epsilon)| \lesssim|\log \epsilon|^{-1}
$$

for $\epsilon<\epsilon_{0},\left|x-x_{0}\right|<\epsilon_{0}$ and $\gamma(x-\epsilon) \in \bigcup_{k=k_{0}}^{\infty} \widetilde{\Lambda}_{k} \cap \Gamma$.

Proof. Let $\epsilon>0$ and $z=\gamma(x) \in \Gamma$. Moreover, let us define $z_{ \pm}:=\gamma(x \pm \epsilon)$. Let $k$ be the maximum index such that $z \in \tilde{\Lambda}_{k}$ and let $k_{ \pm}$be the maximum index such that $z_{ \pm} \in \tilde{\Lambda}_{k_{ \pm}}$. Without loss of generality, we will prove the lemma for $x<x_{0}$. Let us proceed with some geometrical consideration.

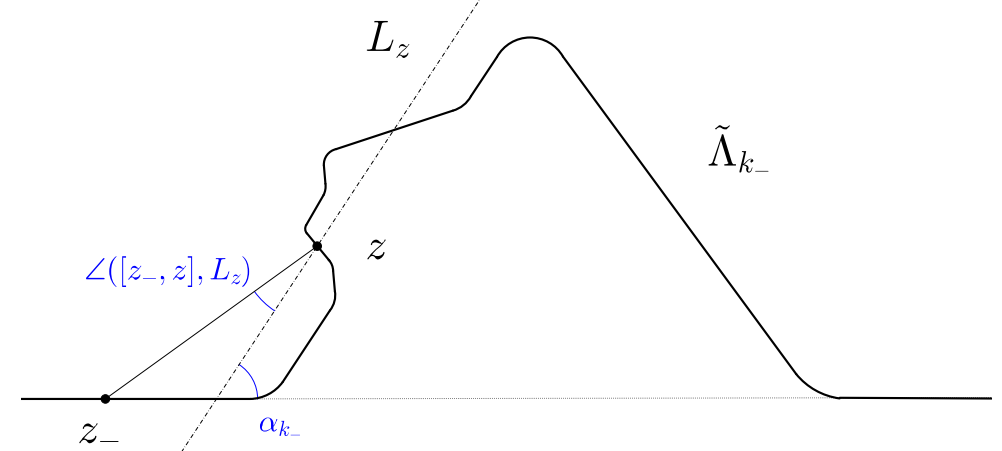

Figure 4. A schematic representation of the setting of the proof of Lemma 9.

Let $L_{z}$ denote the line passing through $z$ and parallel to the segment $\widetilde{I I}_{k_{-}}$. Due to the definition of the angle $\alpha(x, \epsilon)$, we can fix the line $L_{z}$ and bound $|\alpha(x, \epsilon)|$ by the absolute value of the smallest angle $\angle\left(\left[z_{-}, z\right], L_{z}\right)$ that $L_{z}$ forms with the segment $\left[z_{-}, z\right]$ plus the absolute value of the smallest angle $\angle\left(\left[z, z_{+}\right], L_{z}\right)$ that $L_{z}$ forms with the segment $\left[z, z_{+}\right]$.

If $z$ belongs to $\widetilde{\Lambda}_{k_{-}}$, due to the properties of the $\alpha_{k_{-}}$-patch, the arc $\gamma([x-\epsilon, x])$ is entirely contained in a cone of vertex $z$ and aperture $\angle\left(\left[z_{-}, z\right], L_{z}\right)$. By elementary geometric considerations, we can write

$$
\left|\angle\left(\left[z_{-}, z\right], L_{z}\right)\right| \leq \alpha_{k_{-}} .
$$

Again, due to few geometric observations (that are not substantial for the sequel and we decide to omit in order to make the proof more concise) and to the way $\Gamma$ is defined, it is not difficult to see that

$$
\left|\angle\left(\left[z_{+}, z\right], L_{z}\right)\right| \leq 2 \alpha_{k_{-}} .
$$

We are left to consider the case $z \notin \widetilde{\Lambda}_{k_{-}}$. As we observed in Lemma 8 , in this case we have $\left|z_{-}-z\right| \geq L_{k_{-}+1} / 4$. Moreover, $\bigcup_{j=k_{-}+1}^{\infty} \widetilde{\Lambda}_{j} \cap \Gamma$ is contained in a rectangle 
whose base lays on $\widetilde{I I}_{k_{-}}$, whose length is smaller than, say, $5 L_{k_{-}+1} / 3$ and with height $h_{k_{-}+1}$ (for its definition we refer to (5.8) in Lemma 8). We recall that

$$
\frac{h_{j}}{L_{j}} \rightarrow 0 \text { for } j \rightarrow \infty \text {. }
$$

Now observe that $z_{+} \in \bigcup_{j=k_{-}}^{\infty} \widetilde{\Lambda}_{j} \cap \Gamma$. For every point $z$ in this rectangle, using that $\left|z-z_{+}\right| \gtrsim L_{k_{-}+1}$, it holds that

$$
\left|\angle\left(\left[z_{-}, z\right], L_{z}\right)\right| \lesssim \alpha_{k_{-}}
$$

and

$$
\left|\angle\left(\left[z, z_{+}\right], L_{z}\right)\right| \lesssim \alpha_{k_{-}} .
$$

Joining (5.14),(5.15),(5.16) and (5.17), we get

$$
|\alpha(z, \epsilon)| \lesssim \alpha_{k_{-}} \text {. }
$$

Then, by the construction of $\Gamma$ and the definition of $L_{m}, L_{m+1} / L_{m} \leq 1 / 2$ for every $m$, that by iteration leads to

$$
L_{m} \leq 2^{-m}
$$

Now, if $\gamma(x-\epsilon) \in \widetilde{\Lambda}_{k_{-}}$for $k_{-}$big enough, we have that $\epsilon \lesssim L_{k_{-}}$so that

$$
k_{-} \gtrsim|\log \epsilon|
$$

for $\epsilon$ small enough. So, gathering all the considerations and recalling that $\alpha_{k_{-}}=1 / k_{-}$, we get the desired result.

Lemma 10. There exists $\epsilon_{1}>0$ such that the inequality (5.13) holds for $\epsilon<\epsilon_{1}$.

Proof. Let us consider $z_{1}, z_{2} \in \Gamma$. Let $k_{1}$ be the maximum index such that $z_{1} \in \tilde{\Lambda}_{k_{1}}$ and $k_{2}$ the maximum index such that $z_{2} \in \tilde{\Lambda}_{k_{2}}$. Without loss of generality, $k_{1} \leq k_{2}$ and $\gamma^{-1}\left(z_{1}\right) \leq \gamma^{-1}(\tilde{z})$. The idea is to prove that $C_{\epsilon}^{-1}$ is greater than a quantity which approximates $\cos \alpha_{k_{1}}$. It is convenient to split the study into different cases.

If $k_{1}=k_{2}$ and $\gamma^{-1}\left(z_{2}\right)<\bar{x}$ or $k_{2}=k_{1}+1$ and $z_{2} \in \widetilde{I}_{k_{1}+1}$, then (5.2) gives

$$
\left|z_{1}-z_{2}\right| \geq \cos \alpha_{k_{1}} l\left(z_{1}, z_{2}\right) \text {. }
$$

If $k_{1}=k_{2}$ and $\gamma^{-1}\left(z_{2}\right)>\bar{x}$ or $k_{2}=k_{1}+1$ and $z_{2} \in \widetilde{I V}_{k_{1}+1}$, then we can write

$$
\left|z_{1}-z_{2}\right| \geq \cos \alpha_{k_{1}}\left(l\left(z_{1}, z_{2}\right)-R_{k_{1}+1}\right)=\left(\cos \alpha_{k_{1}}-\cos \alpha_{k_{1}} \frac{R_{k_{1}+1}}{l\left(z_{1}, z_{2}\right)}\right) l\left(z_{1}, z_{2}\right)
$$

and we recall that

$$
\frac{R_{k_{1}+1}}{l\left(z_{1}, z_{2}\right)} \lesssim \frac{R_{k_{1}+1}}{L_{k_{1}+1}} \rightarrow 0 \quad \text { for } \quad k_{1} \rightarrow \infty
$$

In the remaining cases, we know from the proof of Lemma 8 that

$$
\left|z_{1}-z_{2}\right| \geq\left(\cos \alpha_{k_{1}}-\cos \alpha_{k_{1}} \frac{h_{k_{1}+1}}{l\left(z_{1}, z_{2}\right)}-\cos \alpha_{k_{1}} \frac{R_{k_{1}+1}}{l\left(z_{1}, z_{2}\right)}\right) l\left(z_{1}, z_{2}\right),
$$

so that, using the same argument as at the end of the proof of Lemma 9 together with the Taylor expansion for the cosine, the proof is completed.

The two previous lemmas show that the arc-length parametrization $\gamma$ of $\Gamma$ is such that the estimate

holds for every $z \in \Gamma$.

$$
T_{*}(f)(z) \lesssim M^{2}(T f)(z)
$$


Final remarks on the curve $\Gamma$. The curve $\Gamma$ that we studied in this section can be considered as an example of a critical curve for which the main theorem holds. Indeed, another look at the estimates we got tells that most of those concerning the geometry of the curve are close to being sharp. Moreover, the finite second difference $|\gamma(x+\epsilon)+\gamma(x-\epsilon)-2 \gamma(x)|$ has the right decay we need; the choice of a slower decay for the angles $\alpha_{j}$ causes worse estimates for $|\alpha(x, \epsilon)|$ and, hence, the finite second difference estimate to fail. Let us notice that the spiraling of $\Gamma$ close to the point $z_{0}$ also gives an idea of how the critical curves may look like.

Asymptotically smooth curves that are not $C^{1}$ may also be defined by means of complex analysis (exploiting, for example, the results in [6]) but we found a constructive approach more convenient to our purposes.

Acknowledgments. The author is thankful to his advisors Xavier Tolsa and Joan Verdera for their valuable help and to the anonymous referee for his/her careful reading of the paper and all the suggestions. This study was partially supported by MTM-2016-77635-P, MDM-2014-044 (MICINN, Spain), 2017-SGR-395 (Catalonia), and by Marie Curie ITN MAnET (FP7-607647).

\section{References}

[1] FalconeR, K.: Fractal geometry: mathematical foundations and applications. - J. Wiley \& sons, 1990.

[2] Girela Sarrión, D.: Counterexamples to some pointwise estimates of the maximal Cauchy transform in terms of the Cauchy transform. - Ann. Acad. Sci. Fenn. Math. 38, 2013, 657-675.

[3] Mateu, J., J. Orobitg, C. PÉrez, and J. Verdera: New estimates for the maximal singular integral. - Int. Math. Res. Not. IMRN 19, 2010, 3658-3722.

[4] Mateu, J., J. Orobitg, and J. Verdera: Estimates for the maximal singular integral: the case of even kernels. - Ann. of Math. (2) 174:3, 2011, 1429-1483.

[5] Pommerenke, Ch.: Boundary behavior of conformal maps. - Springer-Verlag, Berlin, 1992.

[6] Pommerenke, Ch.: On univalent functions, Bloch functions and VMOA. - Math. Ann. 236, 1978, 199-208.

[7] TukiA, P.: The planar Schönflies theorem for Lipschitz maps. - Ann. Acad. Sci. Fenn. Ser. A I Math. 5, 1980, 49-72.

[8] Tukia, P.: Extension of quasisymmetric and Lipschitz embeddings of the real line into the plane. - Ann. Acad. Sci. Fenn. Ser. A I Math. 6, 1981, 89-94.

[9] VerderA, J.: The maximal singular integral: estimates in terms of the singular integral. Proceedings of the XXXI Conference in Harmonic Analysis, Rome, Springer Verlag, 2011.

[10] Stein, E. M.: Singular integrals and differentiability properties of functions. - Princeton Univ. Press, 1970.

[11] Weiss, M., and A. Zygmund: A note on smooth functions. - Nederl. Akad. Wetensch. Proc. Ser. A 62 = Indag. Math. 21, 1959, 52-58.

Received 28 November 2018 • Accepted 18 April 2018 